\title{
A Perspective on the Language-game Rules and Complete Translation Strategy of EST: English for Textile Science and Technology
}

\author{
Hua $\mathrm{Xu}$ \\ Wuhan Polytechnic \\ Wuhan, China 430074
}

\begin{abstract}
Linguistic philosophy is the most important theory source in the study of linguistics. A language-game is a famous philosophical concept developed by Ludwig Wittgenstein and Friedrich Waismann. Wittgenstein's late philosophy theories focus on the essence of language function-communication, i.e. the famous language-game theory which emphasizes the uncertainty of language. The thesis explicates the basic concept of language-game in translation, which guides the translation practice on English for Textile Science and Technology by combining the Game Rules with Complete Translation Strategy.
\end{abstract}

Keywords-language-game; game rules; communication; translation; EST (English for Science and Technology); faithfulness; fluency; complete translation strategy

\section{INTRODUCTION}

Ludwig Wittgenstein, a world-renowned Austrian-born British philosopher of the 20th century, made a general statement about language-game in his "Philosophical Studies": "I also refer to the whole composed of the interweaving of language and activities as 'Languagegame"". [1] The purpose of language gamification is to illustrate the functional essence of language-communication. Language without communication is meaningless, and so is the semantic without communication. Wittgenstein examined the meaning in communication and studied language in communication, so he vividly called it "Language-game". "Language-game Theory" is one of the most important concepts in Wittgenstein's later philosophical views. This theory affects the development of language, including translation. Translation, on the other hand, is an interlingual language-game, which is the basic idea of the functional view of translation and the guiding principle of our translation teaching thoughts. When the translator is faced with the conversion of two languages, the first thing to be determined is what the source language means on earth. Therefore, Wittgenstein said that "to grasp certainty is a language-game”. [1]

The concept of "game" also suggests that participants must follow the rules. In China, many theorists mainly understand the theoretical game concept from the perspective of Game Rules. The concept of games shows that, on the one hand, games are free and have no purpose constraints; on the other hand, all games must follow certain rules. The constraints of game do not come from cause and effect, but from norms. The concept of language-game emphasized by post-Wittgenstein essentially emphasizes the openness of language itself, that is, uncertainty. The use of language does not have a completely closed boundary, but should be determined by context. Language games, like games, have their own rules. Only by obeying the rules in a specific context can we truly use the essential features of language, use language correctly, and achieve the purpose of communication.

\section{CHARACTERISTICS OF EST TRANSLATION AND ITS GAME RULES}

Like other language games, all forms of translation belong to human interlingual communication activities, which is practical and experiential. Translation is different from other forms of language games in that "the main axis and goal throughout the whole activity process are the three parties of the game participants, involving interlingual multidimension and multi-level — interlingual communication and form operation among readers, translators and original authors. Translation language-game, an interlingual practice activity characterized by meaning communication, determines that translatology is an empirical science closely related to human cognitive behavior. [2] Translation standards are the "Game Rules" applicable to all translation practices. Translation standards are related to the internal laws of translation. The former depends on the latter, and the two are external and internal relations. In modern translation theory, "faithfulness" and "fluency" have become the recognized translation standards. [3] The basic feature of translation theory is the cognitive description of experience from the lowest level (translation operation) to the highest level (translation thought). From this we can realize that translatology belongs to applied linguistics and has extensive similarities with other disciplines of language application. Translation studies must have the holism of applied description. [2]

\section{A. Characteristics of EST Translation}

In narrow sense, EST translation is defined as the translation in the field of natural science and engineering 
technology. It studies various substances and phenomena in nature and the experience and knowledge accumulated in their utilization and transformation.[4] Professor Yan Wenpei pointed out that "the purpose of EST translation is to convert the source language text with science and technology and academic content into the target language text through translation, especially the conversion of a foreign language into a mother tongue, so that people who do not understand the original language can learn and understand the academic content of science and technology in the original text through the translation."'[5]

- EST has its own characteristics in addition to the common features of ordinary English. As the carrier of modern science and technology, science and technology works are mainly narrative reasoning, description phenomenon, derivation formula, demonstration law, etc. Therefore, EST's style is dominated by argumentative essay and expository writing that are normative, natural, well-chosen, and rarely rhetorical. Its writing is usually very objective, precise, clear, rigorous, and concise. In addition, EST rarely contains the cultural tradition with unique British and American national characteristics, that is, in terms of word-selection and sentence-construction, EST rarely adopts the expression method rich in cultural metaphor, preferring to use direct and clear statement, which is much more serious than humorous. Therefore, in the use of English to describe or demonstrate science and technology, we must adhere to an objective and fair attitude, and strive to accurately reflect the objective reality. Language expression also requires clear concepts, precise data, rigorous logic and rigorous thinking. Only in this way can we fully embody the spirit of science and reveal the truth of science.

- EST also differs greatly from ordinary English in tense, voice, word order and sentence structure, which is determined by the nature of science and technology. EST usually narrates objective things, describes their occurrence, development and change process, expresses the laws and relations of objective things, and explores and verifies the facts and methods, performance and characteristics, etc. Thus, we should try to avoid the use of "person sentence" causing subjective speculation easily. On the premise of not affecting the accurate and clear expression of the technical content, completely eradicate unnecessary repetition as far as possible, omit as much as possible, and make EST clear and concise. EST's rigorous logic and strict exposition are often achieved through the use of long sentences with complex syntactic structures and tight semantic connections. Hence, EST mostly adopts passive voice, elliptical structure, some idiomatic sentence structure, tense form and long sentence structure. These characteristics reflect the rigorous scientific attitude of scientists and the objective and true expression to scientific facts.
- As far as rhetoric of EST is concerned, besides using a large number of professional terms, it also has the characteristics of nominalization structure of verbs, non-predicate verbs, part of speech conversion, compound words and acronyms. In EST, nouns and nominalization structure of verbs and the nonpredicate verb as attributive are widely used to restrict or replace clauses, so as to meet the requirements of objectivity, accuracy, conciseness, compactness and clearness in scientific and technological style. The conversion of part of speech in EST can be seen everywhere. The most common is the direct conversion of a noun into an adjective, used as a prepositive attribute to modify a noun. The rapid development of science and technology and the emergence of new things and concepts have led to an endless stream of new vocabulary, terms and expressions. While there are three ways to create new words in English: making new words, adding new meanings to old words and recombining old words. Acronyms are the products of people's continuous simplification of words along with the acceleration of life rhythm, and they can be found everywhere in EST literature.

\section{B. The Game Rules of EST Translation}

All the rules in the game of language translation are dialectical. Any game has rules, rules regulate the game activities, and "Language-game" is certainly no exception. There are rules to be followed, but there is a dialectic of "obeying the rules", which Wittgenstein calls "the natural law governing the game". This dialectic is: both to abide by the rules and to control the rules on the premise of mastering all concerned rules. Therefore, EST translation should reflect its dialectical nature, that is, game participants (original author, translator and reader) must skillfully, autonomously and adroitly master the rules according to circumstances on the premise of obeying the rules, namely, the means and strategies adopted in the translation process. These rules are translation theories. Translation theory has the characteristics of restriction, adaptability and relative reference. In consequence, it is necessary to attach importance to the guidance and applicability of the theory, and at the same time recognize the referential and descriptive nature of the rules of translation behavior. [2] A large number of translation practice shows that the emphasis of "Game Rules" in translation varies with the style of the source language. Due to the particularity of scientific and technological style, the purpose, object and function of translation are different from those of general translation; thus, in the practice of EST translation, when "faithfulness" and "fluency" are embodied in the "Game Rules", each of them has its own characteristics.

- Being faithful to the content of the original text and completely reappearing the connotation and thought of the original text are the core issues in the "Game Rules" of EST translation. The translator must express the content of the original work exactly, completely and scientifically, and must not tamper 
with, distort, omit, add or delete, obscure the content of the original work so as to avoid ambiguity. In addition, the meaning of faithfulness also refers to that the original work should maintain the national style, style of the times, linguistic stylistic style, the author's personal language style and so on. So, on the basis of "faithfulness", "Game Rules" of EST translation should conform to its scientific, logical, correct, strict and professional features.

- EST works have a wide range of professional terms, and the "fluency" of science and technology translation must also be in line with professional expression habits and adopt standardized professional language.

Word-choosing and sentence-making should comply with the grammatical rules and writing habits of the target language, strive to be fluent and easy to understand, without any solecism and ungrammatical and incoherent language, mechanically copying, confusing structure, illegibility of logic, word-forword translation, avoid translationese or translation syndrome, and accurately convey modality, tense, voice of the source language, etc. The translated language must be a modern language that conforms to the national language habits and scientific norms. Famous translator Mr. Fu Lei pointed out: the ideal translation seems to be the original author's writing in Chinese. Overseas translation Master Mr. Qian Gechuan also pointed out: the translation must be translated as fluent as creation. It can be seen that translation masters equate translation with Chinese writing. The two Game Rules, "faithfulness" and "fluency", cannot be seen in isolation. They are both independent and unified. They are dialectical and unified relations and are two aspects of a problem. The requirements for "faithfulness" and "fluency" should be reflected in the whole process of translation. In general, accuracy is the main requirement of translation, and on the basis of accuracy, fluency is sought. The scientific and technological articles are accurate and systematic expositions of scientific and technological issues, involving scientific research and production processes, and have a particularly strict requirement on accuracy. [6]

\section{ApPliCATION OF COMPLETE TRANSLATION STRATEGY IN TRANSLATION PRACTICE OF ENGLISH FOR TEXTILE SCIENCE AND TECHNOLOGY}

The process of translation practice is the process of correctly understanding the original text and creatively using the target language to reproduce the original text. Generally, it can be divided into three stages: understanding, expression and checking. In the translation practice of English for textile science and technology, since textile and clothing are closer to people's life than chemical engineering, machinery, astronomy and other cutting-edge science, when paying attention to following the "Game Rules" of "faithfulness" and "fluency" in translation, we should make it closer to people's living language habits. Translators should first combine people's language description characteristics to textile and clothing, have a thorough understanding of the original work, and then express it exactly in Chinese. Complete Translation is a thinking and interlingual activity in which the translator transforms the original information into the cultural information of the target language in order to obtain similar style. Complete Translation strives to convey the content of the original work completely, take into account the form of the original work, and achieve great similarity in style.[4] The Complete Translation Strategy is a means and strategy to definitely apply the "Game Rules" in translation practice to achieve the best effect, including literal translation strategy and liberal translation strategy. "Literal translation and liberal translation are two aspects of a basic means in translation",[4] and they are two means and strategies that must be implemented under the "Game Rules" of translation.

- Literal translation refers to the translation processing method that, on the premise of conforming to the linguistic norms of the target language, maintains both the content of the original text and the form of the original text as far as possible in the translation. [5] Literal translation is the most effective method when the source language and the target language have the same way of expression.[7] For example, "Fibers contribute to the hand, texture and appearance of fabrics." The phrase "contribute to sth." means "to have an effect on sth." The word "hand" in textile profession means "feel". According to the surface structure of the original sentence, this example sentence is literally translated into: “纤维的种类或品质 会影响到织物的手感、质地和外观” in Chinese, which is both faithful to the original text and easy to read. It should be noted, however, that the literal translation is not a verbatim or hard translation of the original form. For instance, "The open-end spinning does not know this disadvantage."'[8] If the sentence is literally translated into “气流纺纱不知道这个缺点” in Chinese, it loses the meaning that EST translation requires objectivity and accuracy, and violates the "Game Rule" of EST translation, although it is faithful to the meaning and form of the original text. The correct translation should be: “气流纺不存在这个缺点” in Chinese 是指侧重于忠实地传达原文语义特别是隐含的信息 而不拘泥于原文形式的翻译处理方式.

- Liberal translation (Free translation) refers to a translation processing method that focuses on faithfully conveying the meaning of the original text, especially the implied information, rather than rigidly adhering to the original form (i.e., beyond restriction of the original form).[5] When there is no corresponding word of the source language in the target language and literal translation cannot convey the original meaning, liberal translation is an effective method to solve the problem.[7] For example, "Everyone is surrounded by textiles from birth to death."[8] In the dictionary, "surround" means “包围, 围绕" in Chinese. If mechanically used, instead of 
choosing the exact Chinese expression according to the context and the characteristics of the textile profession, the example sentence is literally translated into “每个人从生到死都被纺织品所包围” in Chinese, which is not in line with Chinese customs. Although the content of the original text is preserved, it is hard and obscure to read. If the word "surround" is understood as “离不开” in Chinese, and the phrase "from birth to death" as "- - 生" in Chinese, and the original form is appropriately changed, this sentence can be liberally translated into “每个人一生都离不开纺织 品” in Chinese. Obviously, this translation method not only expresses the content of the original text, but also preserves the metaphor of the original text, and simultaneously follows the "Game Rules" of EST translation. For another example, "We sleep on and under fabrics."[8] If we literally translated the sentence into “我们睡在织物之上和织物之下” in Chinese, which clearly is not in accordance with our Chinese language expression habits. The correct translation should be “我们铺的盖的都是纺织品” in Chinese.

It should be pointed out that in terms of EST translation, on the premise of accurately expressing the content of the original text and not violating Chinese norms, literal translation should be adopted as far as possible, which is conducive to maintaining the original style. Free translation is only used when literal translation cannot accurately and properly convey the ideological content of the original text, that is, "literal translation first, free translation second", which should become a very important basic principle in the translation methodology of EST.[5] Therefore, a principle that Mr. Lu Dianyang once put forward can be applied in EST translation, which is "Translate literally, if possible, or appeal to free translation." [6]

\section{CONCLUSION}

The game focuses on participation and interaction, so all players in the game are equal. Translation is no exception. Game participants (translators, readers) must abide by the relevant rules, and pay attention to the participation and interaction of the game. If the game participants of EST translation language lack the tacit understanding of interaction, it is impossible to carry out effective and fruitful translation activities. Therefore, EST translation language game focuses on equal participation and should give translators the right and power of original creation that equal participants should enjoy. The game of translation between words of any two languages is by no means a simple mechanical conversion or a fixed word "matching". The languages of different cultures and nations have their own characteristics and forms, which are different in vocabulary, grammar, usage and expression. The relationship between literal translation and liberal translation contained in Complete Translation Strategy is both independent and unified. As two interdependent aspects of a whole, it is impossible to completely separate them. Therefore, translators must adopt different strategies and use them flexibly in translation. In short, no matter what translation strategies are adopted, the ultimate goal is to faithfully and smoothly express the ideological content and style of the original text, to achieve the essence of language functioncommunication. In the action game activity of translating, it is particularly important to recognize the nature of language and strictly abide by the "Game Rules" in the "Languagegame" of translation.

\section{REFERENCES}

[1] Wittgenstein "Philosophical Studies" [M] Beijing Commercial Press, 1996

[2] Liu Miqing "Translation Teaching: Practice and Theory" [M] Beijing China Foreign Translation and Publication Corporation, 2007

[3] Zhang Peiji "A Course in English-Chinese Translation" [M] Shanghai Shanghai Foreign Education Press, 1980

[4] Huang Zhonglian "Science Translatology" [M] Beijing China Publishing Group China Foreign Translation and Publication Corporation, 2007

[5] Yan Wenpei "Practical Essentials of English Translation for Science and Technology" [M] Beijing Science Press, 2008

[6] Yang Yue "A study on Practical Translation of English for Science and Technology" [M] Xi'an Xi'an Jiaotong University Press, 2008

[7] Zhang Meifang "Functional Approaches to Translation Studies" [M] Shanghai Shanghai Foreign Education Press, 2005

[8] Luo Jutao "Textile Science Course" Second Edition [M] Beijing China Textile Press, 1997 